\title{
Selbstcheck berufliche Vorsorge (BVG)
}

\section{Profitieren Sie von allen Vorteilen der Ärztevorsorgelösungen?}

Nehmen Sie sich fünf Minuten Zeit für den folgenden BVG-Selbstcheck, und finden Sie heraus, ob Sie über Optimierungspotential in der beruflichen Vorsorge verfügen.

Kennen Sie die FMH Insurance Services Pensionskassenlösungen?

$\bigcirc$ JA $\bigcirc$ NEIN

Unsere Angebote sind auf die Bedürfnisse der Ärzteschaft ausgerichtet. Je nach Angebot profitieren Sie z.B. von tieferen Verwaltungskosten oder von einem höheren Umwandlungssatz. Ein Vergleich lohnt sich jetzt, denn normalerweise können Sie bis zum 30. Juni Ihre Verträge für eine Optimierung kündigen.

Haben Sie Ihr BVG-Einkaufspotential ausgeschöpft?

$\bigcirc$ JA $\bigcirc$ NEIN

BVG-Einkäufe können voll vom steuerbaren Einkommen abgezogen werden. So können je nach Einkommenshöhe und Wohnort Steuereinsparungen von bis zu 45\% des BVG-Einkaufes realisiert werden. Die Einkaufsmöglichkeiten sind jedoch von Kasse zu Kasse unterschiedlich.

Kennen Sie die Leistungen Ihrer Vorsorgelösung, und entsprechen sie Ihrem Bedarf?

$\bigcirc$ JA $\bigcirc$ NEIN

Nur wer seine Vorsorgelösung genau kennt, kann seine Pensionierung optimal planen. Die FMH Insurance Services Berater helfen Ihnen gerne und zeigen Ihnen, welche Leistungen Sie versichert haben und wo Sie allenfalls Deckungslücken aufweisen.

Haben Sie überprüft, ob Ihre BVG-Sparbeiträge erhöht werden können?

O JA O NEIN

Bei einer Erhöhung der BVG-Sparbeiträge werden grundsätzlich auch neue Einkaufspotentiale geschaffen, die Sie zur Steueroptimierung nutzen können. Bei den standeseigenen Versicherungslösungen können Sparbeiträge von bis $25 \%$ des versicherten Lohnes vereinbart werden.

Ist Ihr Ehegatte/Ihre Ehegattin in Ihrer beruflichen Vorsorgelösung mitversichert?

$\bigcirc$ JA $\bigcirc$ NEIN

Der in der Praxis mitarbeitende Ehepartner erhält für seine Arbeit oft keinen Lohn und ist daher auch im BVG nicht mitversichert. Durch das Bezahlen eines Lohnes kann der Ehepartner jedoch auch im BVG mitversichert werden und zudem für sich eine Säule $3 a$ abschliessen.

Benötigen Sie Ihr gesamtes Alterskapital für die Rente?

JA $\bigcirc$ NEIN

Durch die Trennung der obligatorischen und überobligatorischen Vorsorge erhöhen Sie Ihre Flexibilität. Nebst den steuerlichen Vorteilen können Sie so nach der Pensionierung flexibel über Ihr Geld verfügen, während Ihr Grundbedarf nach wie vor durch die Rente aus dem obligatorischen Bereich sichergestellt ist.

Auswertung

$0 \times$ NEIN: Herzlichen Glückwunsch, Sie haben die wichtigsten Optimierungen bereits vorgenommen.

1-6× NEIN: Sie verfügen über zusätzliches Optimierungspotential. Wir empfehlen Ihnen die Beratung durch einen FMH Insurance Services Berater.

\section{Antworttalon}

Bitte einsenden oder per Fax an: 0319595010

Vorname / Name

Adresse

PLZ / Ort

Geburtsdatum

Telefon privat/Geschäft

Beste Zeit für einen Anruf

E-Mail-Adresse

Ich wünsche eine persönliche Beratung. Bitte rufen Sie mich an.

Ich interessiere mich für:
Säule $3 a$
Pensionskasse BVG
Pensionsplanung
Rechtsschutzversicherung
Finanz-/Steuerplanung
Berufshaftpflichtversicherung 